
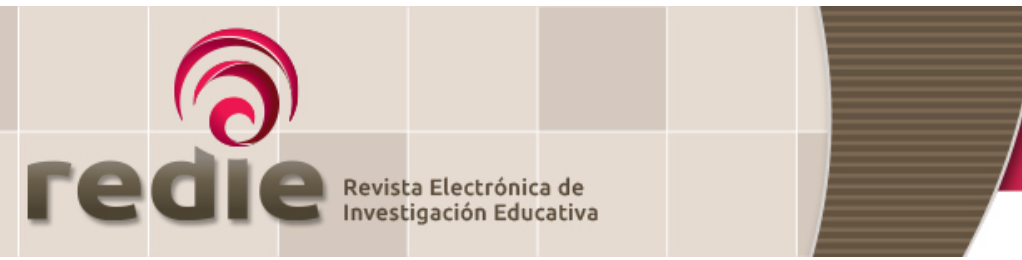

\title{
Aprendizaje colaborativo en línea, una aproximación empírica al discurso socioemocional de los estudiantes
}

\section{Collaborative Online Learning, an Empirical Approach to Students' Socio-Emotional Discourse}

\author{
Juan Carlos Castellanos Ramírez (*) https://orcid.org/0000-0002-0682-9085 \\ Shamaly Alheli Niño Carrasco (*) https://orcid.org/0000-0002-5623-4418 \\ (*) Universidad Autónoma de Baja California \\ (Recibido: 20 de marzo de 2018; Aceptado para su publicación: 17 de julio de 2018) \\ Cómo citar: Castellanos, J. C. y Niño, S. A. (2020). Aprendizaje colaborativo en línea, una aproximación empírica al discurso \\ socioemocional de los estudiantes. Revista Electrónica de Investigación Educativa, 22, e20, 1-12. \\ https://doi.org/10.24320/redie.2020.22.e20.2329
}

\section{Resumen}

Este trabajo presenta una investigación sobre las estrategias discursivas socioemocionales que los estudiantes utilizan durante la elaboración de tareas colaborativas en línea. Se realizó un estudio de casos múltiples para explorar los procesos colaborativos desarrollados por tres grupos de estudiantes inscritos en el primer ciclo académico 2014 de la carrera de psicopedagogía, en la Universitat Oberta de Catalunya. Para resolver la tarea, los estudiantes realizaron contribuciones en un foro asíncrono durante treinta días. Los resultados sugieren que cuatro tipos de estrategias discursivas socioemocionales tienen un efecto importante en el rendimiento de los grupos: i) utilización de pronombres inclusivos, ii) formulación de expectativas sobre la tarea, iii) valoraciones positivas sobre el funcionamiento del grupo y iv) expresiones de apoyo mutuo entre los participantes. También se concluye que los grupos con mejores resultados en su aprendizaje suelen mantener o incrementar el uso de estrategias discursivas socioemocionales a través del tiempo.

Palabras clave: Aprendizaje asistido por computadora, educación superior, trabajo grupal, motivación estudiantil.

\begin{abstract}
This paper presents an analysis of socio-emotional discursive strategies employed by students in collaborative tasks online. A multiple-case study was conducted to explore collaborative processes among three groups of students enrolled in the psychopedagogy degree in the first academic cycle of 2014 at the Open University of Catalonia (UOC). To complete the task, students made contributions to an asynchronous communication forum for thirty days. The results point to four types of socio-emotional discursive strategies that have a significant impact on group performance: i) the use of inclusive pronouns; ii) the formulation of expectations about the task; iii) positive feedback on the way the group is working; and iv) expressions of mutual support among participants. It is also concluded that the groups with the best learning outcomes tend to maintain or increase their use of socio-emotional discursive strategies over time.
\end{abstract}




\section{Introducción}

El aprendizaje colaborativo como objeto de investigación y los beneficios que dichas formas de trabajo producen sobre los aprendizajes de los estudiantes tiene una larga tradición dentro de las ciencias de la educación; el abordaje empírico en torno a dicha temática se ha desarrollado, por ejemplo, desde el campo de la psicología educativa, la pedagogía, la didáctica y el currículum, entre otros (Castellanos et al., 2016; Kyndt et al., 2013; Retnowati et al., 2017).

Las primeras investigaciones realizadas en este campo del conocimiento se centraron en valorar en qué medida y bajo qué circunstancias la realización de tareas grupales producía mejores resultados de aprendizaje en los estudiantes, en comparación con otras formas de organización y actividades en el aula (Dillenbourg et al., 1996). En los últimos 20 años, el foco de estudio pasó de centrarse en analizar el efecto de la colaboración de los estudiantes sobre su rendimiento individual a profundizar en las características de los procesos interactivos y las herramientas discursivas que los estudiantes utilizan en dichas situaciones de aprendizaje (Jeong y Hmelo-Silver, 2016; Malmberg et al., 2015).

Aunado a lo anterior, y debido al rápido desarrollo de las Tecnologías de la Información y la Comunicación (TIC), ha emergido un fuerte interés por el estudio del aprendizaje colaborativo en línea o Computer Supported Colaborative Learning (CSCL) (Jeong y Hmelo-Silver, 2016; Kirschner y Erkens, 2013; Roschelle, 2013), cuyo propósito es explorar la manera en que los estudiantes aprenden a compartir y negociar significados a través de las computadoras conectadas en red.

En este contexto, la mayoría de las investigaciones en CSCL se han centrado en explicar los procesos de construcción compartida del conocimiento a partir del análisis de la evolución de las ideas de los participantes en dichos entornos (Jeong y Hmelo-Silver, 2016; Schwarz et al., 2015), mientras que otras variables relacionadas con el desempeño de los grupos han recibido menor atención por parte de la comunidad científica.

De acuerdo con Colomina y Remesal (2015), Jarvenoja et al. (en prensa), Schoor y Bannert (2011) y Zschocke et al. (2016), una de las cuestiones poco desarrolladas en el ámbito de CSCL son los aspectos motivacionales y afectivos que surgen entre los estudiantes durante su colaboración. Si bien es cierto, aunque hay una fuerte cantidad de investigaciones centradas en el estudio de los componentes motivacionales implicados en el aprendizaje (Del Favero et al., 2007; Elliot et al., 1999; Pajares, 1996), la mayoría de estos trabajos se han realizado en contextos educativos presenciales y tareas de carácter estrictamente individual (Pintrich, 2000; Winne y Perry, 2000; Zimmerman, 2000), mientras que en el campo de CSCL se desconoce aún la influencia que estos aspectos pueden llegar a tener sobre el rendimiento de los grupos. Como destacan Schoor y Bannert (2011): "We possess a large body of research from different traditions showing that motivation plays an important role for individual learning... However, research on motivation influences on CSCL is rare and inconsistent". (p. 560).

En la misma línea, siguiendo los planteamientos de Colomina y Remesal (2015), Zschocke et al. (2016) y Bakhtiar et al. (2018), este trabajo resalta la idea de que el aprendizaje colaborativo es un proceso complejo donde convergen dimensiones sociocognitivas y socioemocionales; por lo tanto, la realización de tareas grupales implica que los estudiantes, además de regular el discurso cognitivo sobre los contenidos temáticos, construyan un contexto socioemocional agradable que les permita una comunicación abierta y constructiva sobre los significados de la tarea. En palabras de Kreijns et al. (2013): "The social interaction should be directed not only toward cognitive processes but also toward socioemotional processes that underlie these cognitive processes; hence, social interaction has both a cognitive dimension and a socioemotional dimension". (p. 230).

En efecto, el interés de nuestro trabajo consiste en explorar cómo los estudiantes regulan y mantienen el contexto socioemocional durante la elaboración de tareas colaborativas en línea. Son tres preguntas las que orientan el presente estudio: 1) ¿Qué estrategias discursivas utilizan los grupos de estudiantes para gestionar sus relaciones socioemocionales durante la realización de tareas colaborativas en línea?; 2) ¿Cómo y de qué manera los grupos de estudiantes sostienen sus relaciones socioemocionales a través del 
tiempo?; 3) ¿Existe alguna relación entre el contexto socioemocional generado por los estudiantes durante la colaboración y el nivel de rendimiento obtenido por los grupos sobre la tarea académica?

\section{Método}

El método utilizado para esta investigación corresponde a un estudio de casos múltiples (Yin, 2006) en el que participaron tres grupos de estudiantes de la carrera de Psicopedagogía, en la Universitat Oberta de Catalunya (UOC), durante el curso académico marzo 2014-junio 2014. En el contexto de nuestro trabajo, cada grupo de estudiantes se consideró un caso de estudio distinto en el entendido de que cada grupo constituye una unidad de análisis diferente y con características específicas. El estudio de casos múltiples nos permite, por una parte, analizar de manera detallada los rasgos socioemocionales propios de cada grupo y, por otra parte, contrastar los resultados entre grupos, obteniendo con ello una mayor robustez y consistencia en las conclusiones (Yin, 2009).

La estrategia metodológica utilizada para la observación de datos se enmarca en el paradigma cualitativo de análisis de la interacción (Garrison et al., 2006). El análisis de la interacción consiste en analizar los mensajes aportados por los estudiantes durante su colaboración e interpretarlos a partir de una teoría definida en torno a los procesos que se investigan (análisis deductivo), esto no excluye que a medida que los investigadores profundizan en los datos se identifican nuevos elementos dentro de la interacción de los estudiantes que obligan a replantear, precisar o cambiar los códigos iniciales (análisis inductivo).

Los grupos analizados se conformaron por cuatro estudiantes que se reunieron bajo acuerdo de afinidad de intereses académicos y relaciones de amistad; en su composición no hubo ningún tipo de intervención directa o indirecta por parte de los investigadores y profesores. Cabe recalcar que, al interior, los grupos de estudiantes presentaban diversidad en cuanto a género y niveles de rendimiento sobre la tarea.

Los grupos trabajaron durante cuatro semanas en la resolución de problemas complejos de asesoramiento educativo. La tarea consistió en que los estudiantes analizaran el ejercicio profesional de asesores expertos y, a partir de ello, propusieran estrategias para mejorar la intervención del asesor. La actividad se llevó a cabo en el campus virtual de la UOC, a través de foros de comunicación asíncrona. Dichos foros se constituyeron como espacios de trabajo a los que sólo tenían acceso los integrantes/miembros de cada pequeño grupo y el profesor de la asignatura.

\subsection{Recolección de datos}

Para acceder a los datos se requirió el permiso del coordinador del programa educativo y luego se realizaron reuniones con los profesores responsables de los grupos para explicarles la finalidad del estudio; por último, se informó a los estudiantes sobre las características del estudio y se les pidió su consentimiento para acceder a la información acumulada en los foros. Según lo descrito, la participación de todas las personas involucradas en la investigación fue voluntaria.

Con la intención de no interferir en el desarrollo natural de las actividades de los estudiantes, accedimos al contenido de los foros una vez que los grupos concluyeron la tarea; en total, se recogieron 382 mensajes/contribuciones realizadas por los grupos.

Los datos recogidos se vaciaron en diversas plantillas elaboradas en Excel y Word, respetando el orden cronológico de los mensajes de los estudiantes. Dichas plantillas constituyen documentos primarios utilizados por los investigadores para una primera examinación analítica.

\subsection{Análisis y codificación de datos}

Con el fin de analizar los procesos colaborativos de los estudiantes se utilizó el programa de análisis cualitativo Atlas.ti para asociar fragmentos del discurso de los estudiantes con distintas categorías y códigos socioemocionales. A continuación se describe el procedimiento de análisis: 
A partir de un proceso iterativo de ida y vuelta entre teoría y datos se fueron estableciendo y refinando los criterios para la codificación del material (contribuciones de los estudiantes). En esta etapa del análisis los investigadores se reunieron dos veces por semana, durante un mes, para revisar de manera conjunta el 50\% del material objeto de análisis. Cabe destacar que el protocolo de codificación se inspiró inicialmente en las propuestas teóricas de presencia social realizadas por Colomina y Remesal (2015) y Zschocke et al. (2016).

Con el propósito de afianzar y dar consistencia interna a los códigos propuestos, se dejó reposar el material por un mes; después de dicho período, los investigadores volvieron a revisar el mismo material y aplicaron los códigos nuevamente. Como resultado de este proceso se comprobó una persistencia en la interpretación de los investigadores sobre el material codificado.

Para la codificación del $50 \%$ del material restante, los investigadores trabajaron de manera individual durante un mes; transcurrido este período, los investigadores volvieron a reunirse para contrastar los resultados. Cuando se presentaban discrepancias sobre la asignación de los códigos, los investigadores revisaban conjuntamente el material y discutían la interpretación del mismo hasta alcanzar el acuerdo. En la tabla I se presenta la lista de categorías y códigos elaborados para el análisis de los grupos.

Tabla I. Protocolo para la codificación del contenido de los foros

\begin{tabular}{|c|c|c|}
\hline Categoría & Código & Descripción \\
\hline \multirow{2}{*}{$\begin{array}{l}\text { Cohesión del } \\
\text { grupo }\end{array}$} & UM_Co1 & Emplean expresiones de cordialidad social. \\
\hline & UM_Co2 & $\begin{array}{l}\text { Emplean pronombres inclusivos que transmiten que el grupo funciona como } \\
\text { equipo y no como agentes individuales. }\end{array}$ \\
\hline \multirow{2}{*}{ Expectativas } & UM_Ex1 & $\begin{array}{l}\text { Crean expectativas positivas en relación con el desempeño personal y/o el } \\
\text { funcionamiento global como grupo. }\end{array}$ \\
\hline & UM_Ex2 & $\begin{array}{l}\text { Crean expectativas positivas sobre el desarrollo o ejecución global de la tarea y/o } \\
\text { los productos elaborados como parte de la tarea. }\end{array}$ \\
\hline \multirow{3}{*}{$\begin{array}{l}\text { Valoraciones } \\
\text { positivas }\end{array}$} & UM_Vr1 & $\begin{array}{l}\text { Realizan estimaciones sobre las exigencias y el esfuerzo que implica la realización } \\
\text { grupal de la tarea. }\end{array}$ \\
\hline & UM_Vr2 & $\begin{array}{l}\text { Estiman positivamente el progreso de la tarea y producto final elaborado } \\
\text { grupalmente. }\end{array}$ \\
\hline & UM_Vr3 & Estiman positivamente el esfuerzo realizado por sus compañeros y el grupo. \\
\hline \multirow{8}{*}{$\begin{array}{l}\text { Componentes } \\
\text { afectivos }\end{array}$} & UM_Em1 & $\begin{array}{l}\text { Comparten emociones vinculadas con experiencias previas a la realización de la } \\
\text { tarea. }\end{array}$ \\
\hline & UM_Em2 & $\begin{array}{l}\text { Expresan emociones o estados de ánimo relacionados con la tarea actual o la } \\
\text { asignatura. }\end{array}$ \\
\hline & UM_Em3 & $\begin{array}{l}\text { Comparten emociones sobre sucesos personales (no relacionados con la } \\
\text { asignatura, tarea, o grupo). }\end{array}$ \\
\hline & UM_Em4 & Recurren al humor mientras tratan asuntos relacionados con la tarea. \\
\hline & UM_Em5 & Palabras de apoyo hacia sus compañeros para la vivacidad de la tarea. \\
\hline & UM_Em6 & Expresan buenos deseos e interés por sus compañeros. \\
\hline & UM_Em7 & $\begin{array}{l}\text { Comparten experiencias o emociones relacionadas con el funcionamiento del } \\
\text { grupo. }\end{array}$ \\
\hline & UM_Em8 & $\begin{array}{l}\text { Controlan estados motivacionales negativos o regulan emociones negativas que } \\
\text { surgen en/entre los miembros del grupo. }\end{array}$ \\
\hline
\end{tabular}

Cabe resaltar que la unidad de análisis de este trabajo fue el fragmento de mensaje. De acuerdo con el protocolo establecido y la propia unidad de análisis, es posible aplicar distintos códigos a un mismo mensaje, siempre y cuando la naturaleza del contenido del texto remita a más de una dimensión socioemocional.

Para determinar si existe algún tipo de relación entre el rendimiento de los grupos y el contexto socioemocional generado por los estudiantes durante su colaboración, se contrastaron los resultados obtenidos en la codificación con las calificaciones finales obtenidas por los estudiantes. En la tabla II se presenta la rúbrica de evaluación utilizada por el profesor para valorar los trabajos finales y asignar la calificación a los grupos. 
Tabla II. Rúbrica de evaluación para valorar el trabajo final de los grupos

\begin{tabular}{|c|c|c|c|c|}
\hline \multirow{2}{*}{ Aspecto valorado } & \multicolumn{4}{|c|}{ Criterios para la asignación de calificaciones } \\
\hline & A (excelente) & B (notable) & C+ (aprobado) & C-/D (suspendido) \\
\hline $\begin{array}{l}\text { Relevancia de la } \\
\text { información }\end{array}$ & Amplia y muy relevante & $\begin{array}{l}\text { Relevante pero } \\
\text { escasa }\end{array}$ & Poco relevante & $\begin{array}{l}\text { No hay selección de } \\
\text { la información }\end{array}$ \\
\hline $\begin{array}{l}\text { Capacidad de } \\
\text { análisis }\end{array}$ & $\begin{array}{l}\text { Muy buena asociación } \\
\text { de ideas, con } \\
\text { información esencial, } \\
\text { bien seleccionada y } \\
\text { estructurada }\end{array}$ & $\begin{array}{l}\text { Aporta ideas } \\
\text { esenciales bien } \\
\text { seleccionadas y } \\
\text { estructuradas }\end{array}$ & $\begin{array}{l}\text { Aporta ideas } \\
\text { generales }\end{array}$ & $\begin{array}{l}\text { No se han } \\
\text { producido ideas } \\
\text { propias }\end{array}$ \\
\hline $\begin{array}{l}\text { Claridad } \\
\text { conceptual }\end{array}$ & Muy clara & Clara & Poco clara & Confusa \\
\hline $\begin{array}{l}\text { Coherencia entre } \\
\text { las ideas }\end{array}$ & $\begin{array}{l}\text { Correspondencia muy } \\
\text { clara entre los } \\
\text { apartados }\end{array}$ & $\begin{array}{l}\text { Correspondencia } \\
\text { adecuada entre los } \\
\text { apartados }\end{array}$ & $\begin{array}{l}\text { Poca coherencia } \\
\text { entre los } \\
\text { apartados }\end{array}$ & $\begin{array}{l}\text { No hay coherencia } \\
\text { entre los apartados }\end{array}$ \\
\hline
\end{tabular}

\section{Resultados}

\subsection{Estrategias discursivas que utilizan los grupos de estudiantes para regular el contexto socioemocional}

Como se ve en la tabla III, las estrategias discursivas que los grupos de estudiantes utilizaron con mayor frecuencia durante su colaboración se enmarcan en la categoría de "cohesión de grupo" y remiten puntualmente a dos aspectos: 1) UM_Co1, palabras de cordialidad tales como saludos, despedidas y agradecimientos (467 registros) y 2) UM_Co2, el uso de pronombres inclusivos para referirse al grupo como equipo (201 registros). En segundo lugar, se observó una diversidad de estrategias discursivas que contribuyen al clima afectivo de los grupos, en donde sobresalen las locuciones vinculadas con: a) UM_Em2, emociones y estados de ánimo positivos relacionados con la tarea (35 registros); b) UM_Em5, palabras de apoyo hacia sus compañeros para la vivacidad de la tarea (28 registros); y c) UM_Em6, comunicación de buenos deseos e interés por sus compañeros (21 registros).

Con respecto a la categoría de "valoraciones", se observaron principalmente dos tipos de estrategias: 1) UM_Vr2, apreciaciones positivas que realizan los estudiantes sobre el progreso de la tarea y el producto final (23 registros) y 2) UM_Vr3, estimaciones positivas sobre el esfuerzo de sus compañeros y el funcionamiento del grupo (28 registros). Por su parte, las estrategias vinculadas con la declaración de expectativas positivas sobre el funcionamiento del grupo y/o el desarrollo de la tarea (UM_Vr1) aparecieron con baja frecuencia dentro de la interacción de los grupos (ver tabla III). 
Tabla III. Estrategias discusivas utilizadas para la regulación socioemocional

\begin{tabular}{cccccc}
\hline \multirow{2}{*}{ Categorías } & \multirow{2}{*}{ Códigos } & Gr1 & Gr2 & Gr3 & \multirow{2}{*}{ Total } \\
\cline { 3 - 5 } Cohesión del grupo & $\mathbf{f}$ & $\mathbf{f}$ & $\mathbf{f}$ & \\
\cline { 1 - 4 } Expectativas & UM_Co1 & 217 & 118 & 132 & 467 \\
& UM_Co2 & 86 & 83 & 32 & 201 \\
& UM_Ex1 & 4 & 0 & 0 & 4 \\
& UM_Ex2 & 5 & 2 & 0 & 7 \\
Valoraciones & UM_Vr1 & 1 & 4 & 0 & 5 \\
& UM_Vr2 & 10 & 11 & 2 & $\mathbf{2 3}$ \\
& UM_Vr3 & 14 & 10 & 4 & $\mathbf{2 8}$ \\
& UM_Em1 & 6 & 3 & 0 & 9 \\
& UM_Em2 & 26 & 7 & 2 & $\mathbf{3 5}$ \\
& UM_Em3 & 1 & 0 & 0 & 1 \\
Afectos & UM_Em4 & 5 & 3 & 0 & 8 \\
& UM_Em5 & 14 & 14 & 0 & $\mathbf{2 8}$ \\
& UM_Em6 & 13 & 4 & 4 & $\mathbf{2 1}$ \\
& UM_Em7 & 2 & 4 & 3 & 9 \\
& UM_Em8 & 3 & 5 & 1 & 9 \\
\hline
\end{tabular}

Nota: * Referencia al número de estrategias discursivas distintas ejercidas por los grupos e identificadas en el número total de fragmentos codificados.

La mayor cantidad de fragmentos identificados en una amplia diversidad de estrategias discursivas para gestionar las relaciones socioemocionales se observaron en el Gr1, ya que los 407 fragmentos identificados remiten al ejercicio de 15 estrategias diferentes. Aunque en el Gr2 la frecuencia de los fragmentos es menor a la observada en el Gr1 (268), también sobresale por la amplia diversidad de estrategias discursivas ejercidas (13). En contraste con estos grupos, el Gr3 se caracteriza tanto por el bajo número de fragmentos identificados (180) como por el número de estrategias distintas empleadas (8).

\subsection{Evolución temporal del discurso socioemocional utilizado por los grupos}

A continuación se muestra cómo los grupos de estudiantes sostuvieron el ejercicio de las distintas dimensiones socioemocionales a lo largo del desarrollo de la tarea.

Mientras Gr1 y Gr2 fortalecían la cohesión de grupo según avanzaban las semanas para el cumplimiento de la tarea, en el Gr3 ocurría lo contrario; durante las últimas dos semanas, los estudiantes del Gr3 actuaban de manera desarticulada y trasmitían un esfuerzo individual -más que colectivo- sobre la tarea, es decir, sobresalían elocuciones sobre responsabilidades individuales (por ejemplo: "tú debes hacer..." "ya acabé mi propuesta", "necesitas incorporar", etc.), en tanto que el uso de pronombres inclusivos (por ejemplo: nosotros, estamos, debemos, etc.) y palabras cordiales (gracias por los comentarios, buenos días, que tengan buen fin de semana, etc.) disminuyeron con el tiempo (figura 1). 


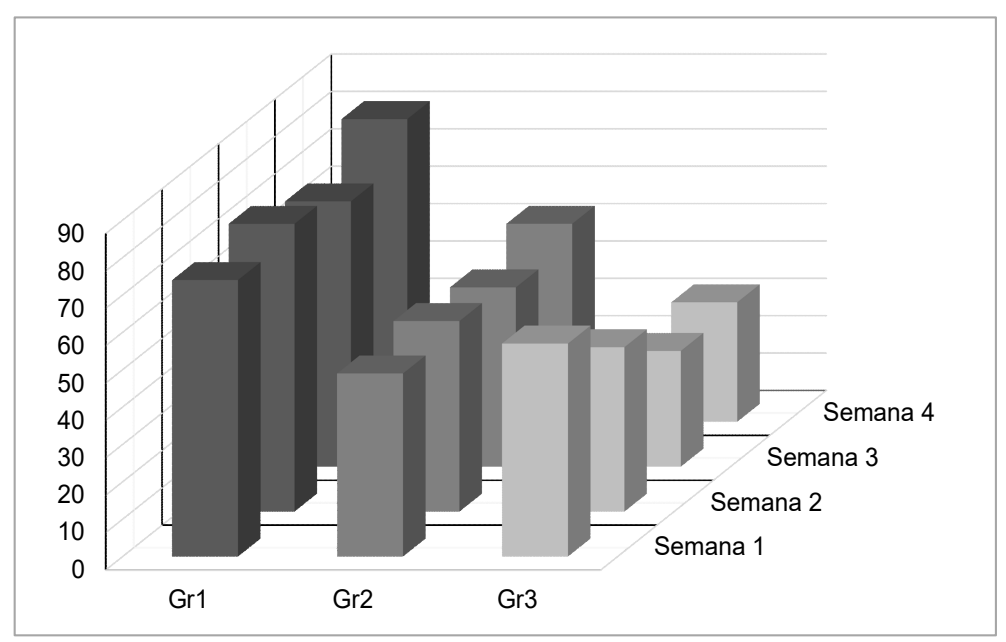

Figura 1. Cohesión de grupo; desarrollo y temporalidad

Como se ve en la figura 2, en el Gr1 las estrategias discursivas que apoyaban el clima afectivo del grupo fueron altas desde el inicio de la tarea y aumentaron conforme avanzaban las semanas. En el Gr2 las estrategias afectivas se utilizaron con poca frecuencia en la parte inicial de la tarea, sin embargo, éstas fueron apareciendo de manera más frecuente en las semanas posteriores; es decir, observamos un desarrollo progresivo de esta dimensión. Por su parte, el Gr3 presentó dificultades para instaurar un clima afectivo favorable desde el inicio de la actividad, tanto así que la presencia de estos componentes decayó entre semana y semana.

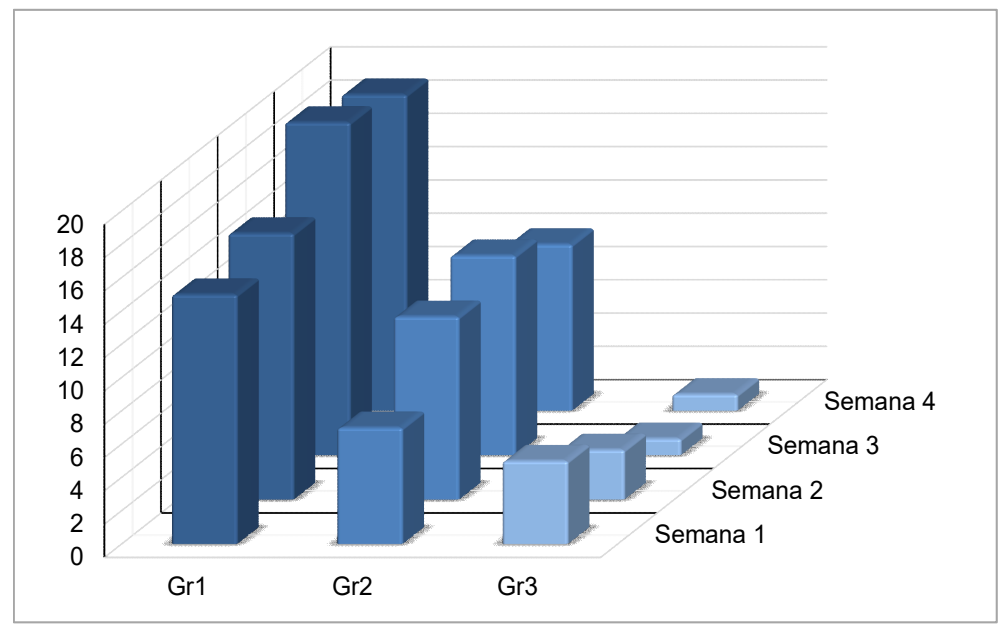

Figura 2. Componentes afectivos; desarrollo y temporalidad

En siguiente figura se observa que las valoraciones positivas sobre la tarea son las estrategias discursivas que los grupos utilizaron con mayor frecuencia en la etapa intermedia de la actividad (semana 2 y 3). Es importante destacar que, en contraste con el Gr3, los grupos Gr1 y Gr2 fueron más constantes en la realización de dichas valoraciones en las cuatro semanas de actividad colaborativa (ver figura 3). 


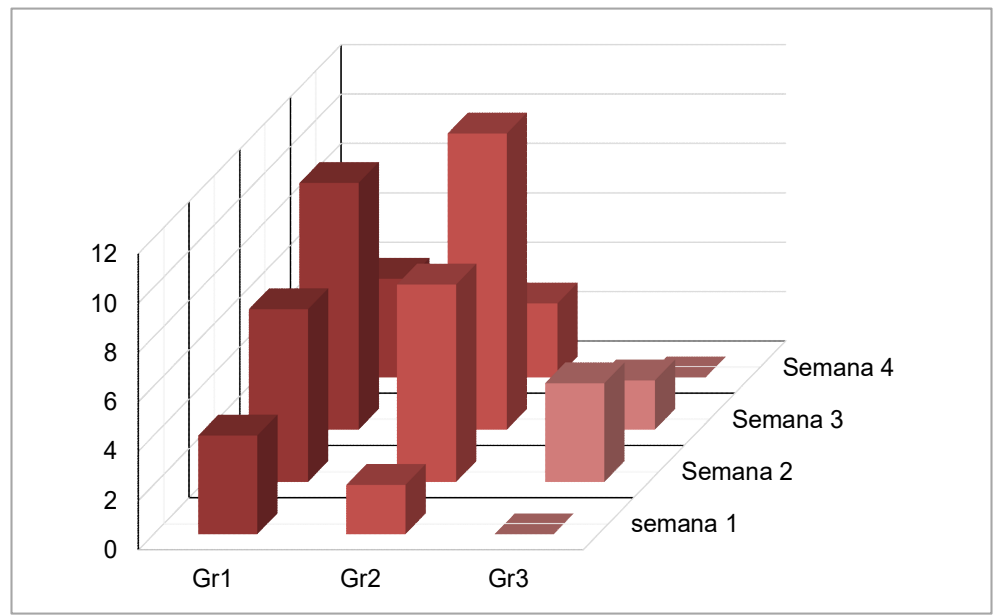

Figura 3. Valoraciones positivas; desarrollo y temporalidad

Como se aprecia en la figura anterior, las valoraciones sobre el desempeño del grupo en la tarea académica son estrategias discursivas que los estudiantes utilizan con poca frecuencia, en comparación con las dimensiones de cohesión de grupo y componentes afectivos.

Por otra parte, según la Figura 4, el planteamiento de expectativas positivas sobre la tarea se realiza en etapas tempranas de la actividad, tal como se observa en los grupos Gr1 y Gr2; en estos casos, dichas estrategias sirven para proyectar las actividades futuras y establecer un primer referente sobre la meta a alcanzar. Por el contrario, puesto que en el Gr3 no se observaron dichas estrategias, los estudiantes de este grupo no plantearon escenarios positivos o direcciones sobre su desempeño grupal.

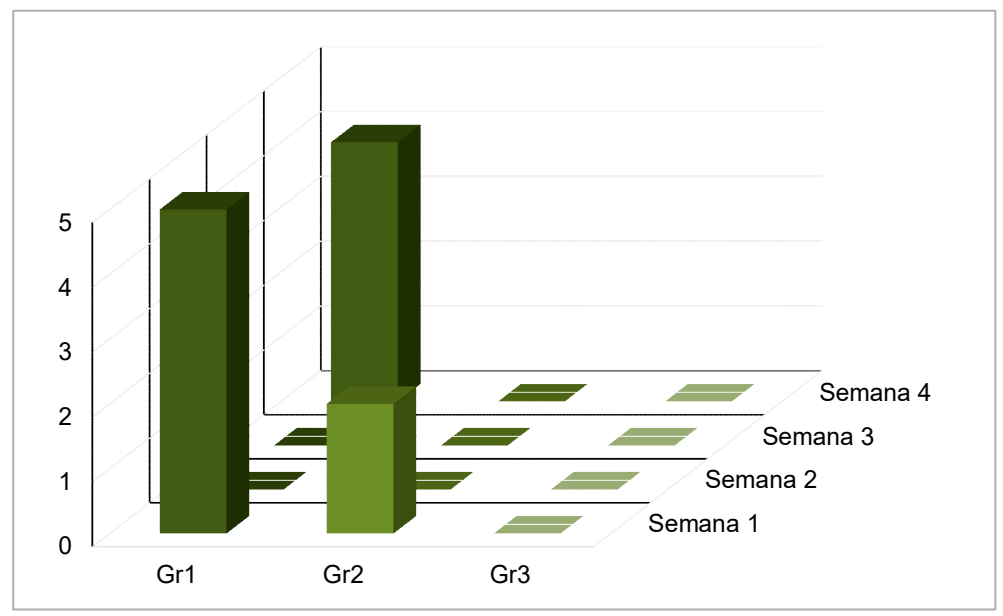

Figura 4. Declaración de expectativas; desarrollo y temporalidad

\subsection{Contraste entre los niveles de rendimiento académico y el discurso socioemocional utilizado por los grupos}

En la tabla IV se muestran las valoraciones realizadas por el profesor sobre los productos finales elaborados por los estudiantes, así como los niveles de rendimiento logrados por los grupos en torno a la tarea académica. 
Tabla IV. Valoraciones otorgadas por el profesor a los productos finales de los estudiantes

\begin{tabular}{|c|c|c|}
\hline Grupos & Valoración de los productos & $\begin{array}{c}\text { Nivel de } \\
\text { rendimiento }\end{array}$ \\
\hline Gr1 & $\begin{array}{l}\text { - Justifican y explican detalladamente los marcos de referencia en que basan sus } \\
\text { propuestas de intervención educativa. } \\
\text { - Los objetivos de sus propuestas son claros en términos conceptuales y } \\
\text { ortográficos. } \\
\text { - Describen las estrategias y criterios de operación para llevar a cabo la intervención } \\
\text { educativa y proporcionan ejemplos para su ejecución. } \\
\text { - El informe final cuenta con índice, paginación, introducción, marco teórico, } \\
\text { propuesta, consideraciones finales, bibliografía y anexos. }\end{array}$ & $\begin{array}{c}\text { A } \\
\text { (excelente) } \\
\text { Alto } \\
\text { rendimiento }\end{array}$ \\
\hline Gr2 & $\begin{array}{l}\text { - Citan los marcos teóricos de referencia en que fundamentan sus propuestas de } \\
\text { intervención pero no desarrollan las ideas a profundidad. } \\
\text { - Algunos objetivos son ambiguos y poco coherentes con el marco teórico en que } \\
\text { fundamentan sus propuestas de intervención. } \\
\text { - Describen las estrategias y criterios de operación para llevar a cabo la intervención } \\
\text { educativa, sin embargo, no dan ejemplos concretos para su ejecución. } \\
\text { - El informe final cuenta con un índice, paginación, marco teórico, propuesta y } \\
\text { bibliografía. } \\
\text {-El grupo no elaboró la introducción del informe, consideraciones finales y anexos. }\end{array}$ & $\begin{array}{c}\text { C+ } \\
\text { (aprobado) } \\
\text { Rendimiento } \\
\text { medio }\end{array}$ \\
\hline Gr3 & $\begin{array}{l}\text { - Las propuestas realizadas no disponen de un marco teórico claro. } \\
\text { - No plantean los objetivos a lograr con el desarrollo de sus propuestas. } \\
\text { - Se plantean las estrategias para desarrollar su intervención educativa sin estipular } \\
\text { los criterios de operación para su aplicación. } \\
\text { - En el informe final hace falta la bibliografía, introducción, consideraciones finales } \\
\text { y anexos. }\end{array}$ & $\begin{array}{c}\text { D } \\
\text { (suspendido) } \\
\text { Bajo } \\
\text { rendimiento }\end{array}$ \\
\hline
\end{tabular}

Según las valoraciones otorgadas por el profesor, hay una relación importante entre el nivel de rendimiento obtenido sobre la tarea académica y el contexto socioemocional generado por los estudiantes durante su colaboración. En efecto, el grupo que alcanzó la calificación más alta en la tarea (Gr1) es el grupo que presenta la mayor cantidad y diversidad de recursos discursivos para gestionar las distintas dimensiones socioemocionales; además, este grupo se caracteriza porque sostuvo la gestión socioemocional y ésta aumentó con el paso del tiempo. Por su parte, el grupo que alcanzó un nivel de rendimiento medio (Gr2) presenta una menor cantidad y diversidad de estrategias socioemocionales; no obstante, la cohesión de grupo y el clima afectivo se mantuvieron favorablemente durante las cuatro semanas de trabajo colaborativo, pero no aumentaron. Por último, el grupo que obtuvo un bajo rendimiento sobre la tarea académica (Gr3) presenta también un clima socioemocional deficiente, ya que tanto la cohesión de grupo, como el clima afectivo, las valoraciones positivas y la declaración de expectativas disminuyeron con el paso del tiempo.

\section{Discusión y conclusiones}

En primer lugar, los resultados de este trabajo apuntan una relación importante entre el discurso socioemocional utilizado por los estudiantes durante su colaboración y el rendimiento grupal obtenido sobre la tarea académica. Este hallazgo refuerza los trabajos de Zschocke et al. (2016) y Jarvenoja et al. (en prensa), quienes también constatan una relación significativa entre el éxito de los grupos y la cantidad de discurso socioemocional utilizado durante la colaboración.

En segundo lugar, se concluye que la dimensión motivacional en el aprendizaje colaborativo tiene un carácter dinámico y evolutivo. De acuerdo con los resultados de este trabajo, para salvaguardar la convivencia y las relaciones afectivas es importante que los estudiantes mantengan un discurso socioemocional positivo a lo largo del tiempo; el uso progresivo en el tiempo de dichas estrategias ayuda a que los estudiantes desarrollen discusiones más profundas y constructivas sobre los contenidos de la tarea. 
En tercer lugar, se observó que las estrategias discursivas socioemocionales que los estudiantes utilizan con mayor frecuencia durante su colaboración en línea remiten a la utilización de un lenguaje inclusivo y al uso de expresiones de cordialidad; estos resultados coinciden con las investigaciones previas de Colomina y Remesal (2015), quienes también remarcaron la importancia de estos dos mecanismos discursivos para el fortalecimiento de la cohesión de grupo. No obstante, nuestros resultados amplían las aportaciones de Colomina y Remesal al precisar que la utilización de un lenguaje inclusivo y las expresiones de cordialidad son especialmente importantes cuando su uso se sostiene y se incrementa de manera progresiva a lo largo de la tarea; de hecho, para que los alumnos mantengan la cohesión del grupo necesitan ser constantes en el uso de tales estrategias, de lo contrario la disminución de dichos mecanismos puede indicar un deterioro en la convivencia del grupo, tal como se observó en el grupo Gr3.

En cuarto lugar, se concluye que los grupos con mayor rendimiento en la tarea son aquellos que emplean una mayor diversidad de estrategias discursivas socioemocionales a lo largo del proceso colaborativo. Las estrategias discursivas que pueden asociarse con un mayor rendimiento en la tarea son: la utilización de pronombres inclusivos para referirse al grupo como equipo, valoraciones positivas sobre el funcionamiento del grupo, así como palabras de apoyo mutuo para impulsar la actividad cognitiva de los participantes. Por lo tanto, de manera similar a los estudios en los que se destaca la importancia de las valoraciones positivas en el aprendizaje individual de los estudiantes (Pintrich, 2000; Winne y Perry, 2000; Zimmerman, 2000), se considera que las autovaloraciones también son determinantes para el buen desempeño de los grupos en las situaciones de aprendizaje colaborativo.

En quinto lugar, los resultados señalan también que aunque la formulación de expectativas sobre la tarea académica es una estrategia discursiva poco frecuente en los grupos, una mínima cantidad de discurso dirigido a este aspecto puede ser suficiente para que los estudiantes encaminen sus esfuerzos hacia una meta clara. Tal como Schoor y Bannert (2011) mencionan, la manifestación de expectativas es una estrategia discursiva que los estudiantes utilizan para proyectar direcciones y metas de aprendizaje; sin ellas, muchas veces los grupos no tienen un rumbo claro sobre cómo y qué se pretende alcanzar. En el marco de esta investigación, constatamos que los estudiantes del grupo que se plantean expectativas sobre la tarea con mayor frecuencia (Gr1) son los que, además de construir productos de mayor calidad, obtienen las mejores calificaciones en la tarea.

A partir de las conclusiones anteriores se subraya una serie de implicaciones para la práctica del profesorado: 1) en entornos de CSCL las ayudas del profesor no deben orientarse sólo al mejoramiento del proceso cognitivo de los estudiantes, sino que también deben ayudar en la instauración de un clima afectivo abierto y flexible entre los estudiantes; 2 ) en etapas tempranas de la colaboración es importante que el profesor ayude a construir una interdependencia positiva entre los participantes animándolos a compartir sus expectativas personales sobre la tarea y, a partir de ello, orientarlos en la formulación de una meta común; 3) en etapas avanzadas de la colaboración se requiere que el profesor estimule al grupo reconociendo aciertos y avances alcanzados en la tarea y exhorte a la culminación de la meta inicial; 4) es importante que el profesor dé seguimiento a las actividades realizadas por los grupos dentro de los foros y, además, permanecer atento a las dificultades o desafíos socioemocionales que pudieran presentarse entre los participantes; cuando los miembros de un grupo no son capaces de regular el contexto motivacional el profesor debe intervenir y sentar las bases de una red afectiva y de confianza entre los participantes.

Considerando los puntos anteriores, futuras investigaciones deberían abordar el papel del profesor como fuente de apoyo a las relaciones socioemocionales de los participantes. Tal como se ha observado en este trabajo, las relaciones socioemocionales son procesos dinámicos que presentan avances y retrocesos a través del tiempo, por lo que su manifestación en los grupos adquiere características diversas; ante estas circunstancias, el profesor necesita ajustar la ayuda educativa considerando las necesidades y características de cada grupo. En este sentido, una línea de investigación abierta consiste en explorar las estrategias que los profesores utilizan para promover relaciones afectivas entres los miembros de un grupo y, además, cómo estas estrategias inciden en la calidad del discurso socioemocional de los estudiantes y en su rendimiento escolar. 


\section{Referencias}

Bakhtiar, A., Webster, E. A. y Hadwin, A. F. (2018). Regulation and socio-emotional interactions in a positive and a negative group climate. Metacognition Learning, 13, 57-90. https://doi.org/10.1007/s11409$\underline{017-9178-x}$

Castellanos, J. C., Onrubia, J. y Niño, S. (2016). El aprendizaje colaborativo mediado por ordenador: avances y desafíos desde una perspectiva psicoeducativa. En E. Román, M. Porras, A. Madrigal y P. Medina (Eds.), Las ciencias de la educación en el proceso de formación del profesional (pp. 70-85). Red Educativa Mundial. https://www.reed-edu.org/wp-content/uploads/2016/12/libro-REED-1.pdf

Colomina, R. y Remesal, A. (2015). Social presence and virtual collaborative learning processes in higher education. Journal for the Study of Education and Development, 38(3), 647-680.

https://doi.org/10.1080/02103702.2015.1054664

Del Favero, L., Boscolo, P., Vidotto, G. y Vicentini, M. (2007). Classroom discussion and individual problemsolving in the teaching of history: do different instructional approaches affect interest in different ways? Learning and Instruction, 17(6), 635-657. https://doi.org/10.1016/j.learninstruc.2007.09.012

Dillenbourg, P., Baker, M., Blaye, A. y O'Malley, C. (1996). The evolution of research on collaborative learning. En E. Spada y P. Reiman (Eds.), Learning in humans and machine: towards an interdisciplinary learning science (pp. 189-211). Elsevier.

Elliot, A., McGregor, H. y Gable, S. (1999). Achievement goals, study strategies, and exam performance: a mediational analysis. Journal of Educational Psychology, 91(3), 549-563. https://doi.org/10.1037/0022$\underline{0663.91 .3 .549}$

Garrison, D. R., Cleveland-Innes, M., Koole, M. y Kappelman, J. (2006). Revisiting methodological issues in transcript analysis of transcripts: negotiated coding and reliability. The internet and higher education, 9(1), 1-8. https://doi.org/10.1016/j.iheduc.2005.11.001

Jarvenoja, H., Jarvela, S. y Malmberg, J. (en prensa). Supporting groups' emotion and motivation regulation during collaborative learning. Learning and Instruction.

https://doi.org/10.1016/j.learninstruc.2017.11.004

Jeong, H. y Hmelo-Silver, C. E. (2016). Seven affordances of computer-supported collaborative learning: how to support collaborative learning? How can technologies help? Educational Psychologist, 51(2), 247265. https://doi.org/10.1080/00461520.2016.1158654

Kirschner, P. A. y Erkens, G. (2013). Toward a framework for CsCL research. Educational Psychologist, 48(1), 1-8. https://doi.org/10.1080/00461520.2012.750227

Kreijns, K., Kirschner, P. A. y Vermeulen, M. (2013). Social aspects of CSCL environments: a research framework. Educational Psychologist, 48(4) 229-242. https://doi.org/10.1080/00461520.2012.750225

Kyndt, E., Raes, E., Lismont, B., Timmers, F., Cascallar, E. y Dochy, F. (2013). A meta-analysis of the effects of face-to-face cooperative learning. Do recent studies falsify or verify earlier findings? Educational Research Review, 10, 133-149. https://doi.org/10.1016/j.edurev.2013.02.002

Malmberg, J., Jarvel a, S., Jarvenoja, H. y Panadero, E. (2015). Promoting socially shared regulation of learning in CSCL: progress of socially shared regulation among high-and low-performing groups. Computers in Human Behavior, 52, 562-572. https://doi.org/10.1016/j.chb.2015.03.082

Pajares, F. (1996). Self-efficacy beliefs in academic settings. Review of Educational Research, 66(4), 543578. https://doi.org/10.3102/00346543066004543 
Pintrich, P. (2000). The role of goal orientation in self-regulation learning. En M. Boakerts, P. Pintrich y M. Zeidner (Eds.), Handbook of self-regulation (pp. 451-502). Academic press. https://doi.org/10.1016/B978$\underline{012109890-2 / 50043-3}$

Retnowati, E., Ayres, P. y Sweller, J. (2017). Can collaborative learning improve the effectiveness of worked examples in learning mathematics? Journal of Educational Psychology, 109(5), 666-679.

https://doi.org/10.1037/edu0000167

Roschelle, J. (2013). Special issue on CSCL: discussion. Educational Psychologist, 48(1), 67-70. https://doi.org/10.1080/00461520.2012.749445

Schoor, C. y Bannert, M. (2011). Motivation in a computer-supported collaborative learning scenario and its impact on learning activities and knowledge acquisition. Learning and Instruction, 21(4), 560-573.

https://doi.org/10.1016/j.learninstruc.2010.11.002

Schwarz, B.B., De-Groot, R., Mavrikis, M. y Dragon, T. (2015). Learning to Learn together with CSCL tools. International Journal of Computer-Supported Collaborative Learning, 10(3), 239-271.

https://doi.org/10.1007/s11412-015-9216-0

Winne, P. y Perry, N. (2000). Measuring self-regulated learning. En M. Boakerts, P. Pintrich y M. Zeidner (Eds.), Handbook of self-regulation (pp. 531-566). Academic press. https://doi.org/10.1016/B978012109890-2/50045-7

Yin, R. K. (2006). Case study methods. En J. L. Green, G. Camilli y P. B. Elmore (Eds.), Handbook of complementary methods in education research (pp. 111-122). L. Erlbaum.

Yin, R. K. (2009). Case study research: design and methods (4a. ed.). Sage.

Zimmerman, B. (2000). Attaining self-regulation: a social cognitive perspective. En M. Boeakaerts, P. Pintrich y M. Zeidner (Eds.), Handbook of self-regulation (pp. 13-39). Academic press.

https://doi.org/10.1016/B978-012109890-2/50031-7

Zschocke, K., Wosnitza, M. y Bürger, K. (2016). Emotions in group work: insights from an appraisaloriented perspective. European Journal of Psychology of Education, 31, 359-384.

https://doi.org/10.1007/s10212-015-0278-1 